\title{
ON TRANSPOSES OF NILPOTENT MATRICES OVER ARBITRARY RINGS
}

\author{
THOMAS P. KEZLAN
}

(Received 5 January 1976; revised 9 September 1976)

\begin{abstract}
It is shown that if every nilpotent $2 \times 2$ matrix over a ring has nilpotent transpose, then the commutator ideal must be contained in the Jacobson radical, thus generalizing a result of $R$. S. Gupta, who considered the division ring case. Moreover, if the nilpotent elements form an ideal or if the ring satisfies a polynomial identity, then the above property of the transpose implies that in fact the commutator ideal must be nil.
\end{abstract}

Gupta (1970) showed that a division ring $D$ must be commutative if every nilpotent $2 \times 2$ matrix over $D$ has nilpotent transpose. Here we show that the same result extends to semisimple rings; that it does not hold for arbitrary rings may be seen by considering a noncommutative nilpotent ring. Thus we turn our attention to the commutator ideal and are able to generalize the above result on semisimple rings by showing that if every nilpotent $2 \times 2$ matrix over a ring $R$ has nilpotent transpose, then the commutator ideal of $R$ is contained in the Jacobson radical. We are unable to show that in fact the commutator ideal must be nil, except in special cases - for example, whenever the nilpotent elements form an ideal, or whenever the ring satisfies a polynomial identity.

We shall use the following notation:

$R_{n}=$ the ring of $n \times n$ matrices over $R$;

$A^{t}=$ the transpose of the matrix $A$ in $R_{n}$;

$C(R)=$ the commutator ideal of $R$, that is, the ideal generated by all commutators $[x, y]=x y-y x$;

$J(R)=$ the Jacobson radical of $R$;

$N(R)=$ the Köthe radical of $R$, that is, the sum of all nil ideals of $R$.

An element $x$ in a ring $R$ will be called left singular if and only if $y x=0$ for some nonzero element $y$ in $R$; otherwise $x$ is called left regular. It will be convenient to label several properties of a ring $R$ as follows: 
(N) Every nilpotent $2 \times 2$ matrix over $R$ has nilpotent transpose.

(S) Every nilpotent $2 \times 2$ matrix over $R$ has left singular transpose.

$\left(N^{\prime}\right)$ For all $x$ and $y$ in $R$ the matrix $\left(\begin{array}{cc}-x y & -y x y \\ x & y x\end{array}\right)$ is nilpotent.

$\left(S^{\prime}\right)$ For all $x$ and $y$ in $R$ the matrix $\left(\begin{array}{cc}-x y & -y x y \\ x & y x\end{array}\right)$ is left singular.

Properties $\left(N^{\prime}\right)$ and $\left(S^{\prime}\right)$ are inspired by the fact, as observed by Gupta, that the matrix $\left(\begin{array}{cc}-x y & x \\ -y x y & y x\end{array}\right)$ is always nilpotent. Thus we have $(N) \Rightarrow(S) \Rightarrow$ $\left(S^{\prime}\right)$ and $(N) \Rightarrow\left(N^{\prime}\right) \Rightarrow\left(S^{\prime}\right)$. Furthermore, property $\left(N^{\prime}\right)$ has the advantage of being inherited by subrings and homomorphic images.

THeorem 1. Suppose $R$ satisfies $\left(S^{\prime}\right)$ and $x$ and $y$ are in $R$. If $x$ is left regular, then $y x^{2} y-x y^{2} x$ is left singular.

Proof. Let $x$ and $y$ be in $R$ with $x$ left regular. If in $\left(S^{\prime}\right)$ we replace $y$ by $x y$, we have that the matrix $\left(\begin{array}{cc}-x^{2} y & -x y x^{2} y \\ x & x y x\end{array}\right)$ is left singular. Hence there exist $c$ and $d$ in $R$, not both 0 , such that

$$
\begin{aligned}
c x^{2} y-d x & =0, \\
c x y x^{2} y-d x y x & =0 .
\end{aligned}
$$

Thus $c x y x^{2} y=d x y x=c x^{2} y^{2} x$, whence

$$
c x\left(y x^{2} y-x y^{2} x\right)=0 .
$$

If $y x^{2} y-x y^{2} x$ is left regular, then $c x=0$ with $x$ left regular and hence $c=0$. But then $d x=0$ and so $d=0$, a contradiction. Thus $y x^{2} y-x y^{2} x$ must be left singular.

Corollary 1. If $R$ satisfies $\left(S^{\prime}\right)$ and has no zero-divisors, then $R$ is commutative.

Proof. It follows from Theorem 1 that $R$ satisfies the polynomial identity $x y^{2} x=y x^{2} y$, and since $R$ is semiprime, it must be commutative by Awtar (1973).

TheOREM 2. If $R$ satisfies $\left(N^{\prime}\right)$, then $C(R) \subset J(R)$.

Proof. If $R$ is a division ring, then Corollary 1 and the fact that $\left(N^{\prime}\right) \Rightarrow\left(S^{\prime}\right)$ imply that $R$ must be commutative. Now suppose that $R$ is primitive but not a division ring. Then there exists a division ring $D$ such that $D_{2}$ inherits property $\left(N^{\prime}\right)$. Using property $\left(N^{\prime}\right)$ with $x=e_{12}$ and $y=e_{21}$ in $D_{2}$, we get 


$$
\begin{aligned}
A & =\left(\begin{array}{cc}
-x y & -y x y \\
x & y x
\end{array}\right)=\left(\begin{array}{rr}
-e_{11} & -e_{21} \\
e_{12} & e_{22}
\end{array}\right), \\
A^{2} & =\left(\begin{array}{cc}
e_{11}-e_{2 t} & 0 \\
0 & e_{22}-e_{11}
\end{array}\right),
\end{aligned}
$$

and $A^{4}=\left(\begin{array}{ll}1 & 0 \\ 0 & 1\end{array}\right)$, contradicting the fact that $A$ must be nilpotent. Thus a primitive ring satisfying $\left(N^{\prime}\right)$ must be commutative. A semisimple ring $R$ satisfying $\left(N^{\prime}\right)$ is a subdirect product of primitive rings, each of which also satisfies $\left(N^{\prime}\right)$ and is therefore commutative; hence $R$ is also commutative. Finally, if $R$ is an arbitrary ring satisfying $\left(N^{\prime}\right)$, then $R / J(R)$ is semisimple and satisfies $\left(N^{\prime}\right)$ and is therefore commutative, whence $C(R) \subset J(R)$.

As mentioned before, we do not know whether property $\left(N^{\prime}\right)$, or even the (apparently) stronger property $(N)$, implies that $C(R)$ is nil. If the nilpotent elements form an ideal however, this implication does hold.

THEOREM 3. If the nilpotent elements of $R$ form an ideal and $R$ satisfies $\left(N^{\prime}\right)$, then $C(R)$ is nil.

Proof. We first consider the case in which $R$ has no nonzero nilpotent elements. By Theorem 2 of Andrunakievic and Rjabuhin (1968), $R$ is a subdirect product of rings $R_{\alpha}$ without zero-divisors. Each $R_{\alpha}$ inherits property $\left(N^{\prime}\right)$ from $R$ and so by Corollary 1 and the fact that $\left(N^{\prime}\right) \Rightarrow\left(S^{\prime}\right)$, each $R_{\alpha}$, and therefore $R$, is commutative.

For general $R$ we let $\bar{R}=R / N(R)$; from the hypothesis $\bar{R}$ has no nonzero nilpotent elements and satisfies $\left(N^{\prime}\right)$, whence $\bar{R}$ is commutative. Thus $C(R) \subset N(R)$ and hence $C(R)$ is nil.

We now determine some classes of rings which necessarily satisfy $(N)$.

Lemma 1. If $R$ is any ring and $A_{1}, \cdots, A_{k}$ are in $R_{n}$, then

$$
\left(A_{1} A_{2} \cdots A_{k}\right)^{t}-A_{k}^{t} \cdots A_{2}^{t} A_{i}^{\prime}
$$

is in $C(R)_{n}$.

Proof. For $n \times n$ matrices $A_{1}, A_{2}, \cdots, A_{k}$ over a commutative ring it is well known that

$$
\left(A_{1} A_{2} \cdots A_{k}\right)^{t}=A_{k}^{\prime} \cdots A_{2}^{i} A_{1}^{\prime}
$$

But $\bar{R}=R / C(R)$ is commutative. Since $R_{n} / C(R)_{n} \cong \bar{R}_{n}$ and the isomorphism preserves transposes, the result follows.

THEOREM 4. If $C(R)_{2}$ is nil, then $R$ satisfies $(N)$. 
Proof. Let $A \in R_{2}$ be nilpotent, say $A^{k}=0$. Using Lemma 1 with $A_{1}=A_{2}=\cdots=A_{k}=A$, we have

$$
\left(A^{t}\right)^{k}=\left(A^{i}\right)^{k}-\left(A^{k}\right)^{t} \in C(R)_{2}
$$

and hence $\left(A^{\prime}\right)^{k}$, and therefore $A^{\prime}$, is nilpotent.

Since a matrix ring over a locally nilpotent ring is locally nilpotent, as a corollary of Theorem 4 we have

Corollary 2. If $C(R)$ is locally nilpotent, then $R$ satisfies $(N)$.

COROLlary 3. If $R$ satisfies a polynomial identity, then $R$ satisfies $(N) \Leftrightarrow$ $R$ satisfies $\left(N^{\prime}\right) \Leftrightarrow C(R)$ is nil.

Proof. We already have $(N) \Rightarrow\left(N^{\prime}\right)$. Suppose $C(R)$ is nil. Then $C(R)$, as a nil P.I.-ring, is locally nilpotent by Jacobson $(1964$, p. 232$)$, and hence $R$ satisfies $(N)$ by Corollary 2 . We now have $C(R)$ nil $\Rightarrow(N) \Rightarrow\left(N^{\prime}\right)$; to complete the proof we assume $R$ satisfies $\left(N^{\prime}\right)$. To show that $C(R)$ is nil, it suffices to assume that $R$ is an algebra over a field, using a standard technique (see, for example, Drazin (1955), Herstein (1963), Kezlan (1966)). Let $c \in C(R)$. Then

$$
c=\sum n_{i} z_{i}+\sum z_{i} r_{i}+\sum s_{i} z_{i}+\sum u_{i} z_{i} v_{i}
$$

where the $n_{i}$ are integers and the $r_{i}, s_{i}, u_{i}, v_{i}, x_{i}$, and $y_{i}$ are in $R$ where $z_{i}=\left[x_{i}, y_{i}\right]$. Let $R_{0}$ be the subalgebra of $R$ generated by the $r_{i}$, si , etc. Then $R_{0}$ is a finitely generated algebra satisfying a polynomial identity, whence $J\left(R_{0}\right)$ is nil by Amitsur (1957). Since $R_{0}$ also satisfies $\left(N^{\prime}\right)$, we have $c \in C\left(R_{0}\right) \subset$ $J\left(R_{0}\right)$ and so $c$ is nilpotent.

To conclude we give an example to show that Theorem 2 cannot be strengthened by replacing $\left(N^{\prime}\right)$ with $\left(S^{\prime}\right)$ or even $(S)$ and that Corollary 3 cannot be strengthened by including either $\left(S^{\prime}\right)$ or $(S)$ as a property equivalent with those given there.

LEMMA 2. The group algebra of a locally finite group over any field satisfies $(S)$.

Proof. Let $G$ be a locally finite group, $F$ a field, and $A \neq 0$ a nilpotent $2 \times 2$ matrix over $F[G]$, say $A^{k}=0$. To show that $A^{t}$ is left singular, it suffices to show that $\left(A^{\prime}\right)^{k}$ is left singular. There exists a finite subgroup $H$ of $G$ such that $A \in(F[H])_{2}$. By Lemma 1 with $A_{1}=A_{2}=\cdots=A_{k}=A$, we get $\left(A^{t}\right)^{k} \in C(F[H])_{2}$. Let $\left(A^{t}\right)^{k}=\left(a_{i j}\right)$ where the $a_{i j}$ are in $C(F[H])$. Then

$$
a_{i j}=\sum r_{i j l} z_{l} s_{i j t}
$$


where each $z_{l}$ is a (ring) commutator of two elements of $H$ and where the $r_{i j l}$ and $s_{i j l}$ are in $F[H]$. Using $h z_{l}=\left[h, z_{l}\right]+z_{l} h$, we may assume without loss of generality that

$$
a_{i j}=\sum z_{i} s_{i j l} .
$$

Let $b=\Sigma_{h \in H} h$; then $b h=h b=b$ for all $h \in H$, whence $b z_{l}=0$ for all $l$. Thus $b a_{i j}=0$ for all $i$ and $j$, and letting $B=b e_{11}$ we have $B\left(A^{i}\right)^{k}=0$ with $B \neq 0$.

Example. Let $G$ be a finite non-Abelian group and $F$ a field of characteristic 0 ; thus $F[G]$ is semisimple by Maschke's Theorem. $F[G]$ satisfies $(S)$ by Lemma 2; however the conclusion of Theorem 2 cannot hold since $C(F[G]) \subset J(F[G])=(0)$ implies $F[G]$ is commutative, a contradiction. Thus we cannot replace $\left(N^{\prime}\right)$ with $(S)$ in Theorem 2 . Moreover, $F[G]$, being a finite-dimensional algebra over a field, satisfies a polynomial identity; however $F[G]$ satisfies $(S)$ but not $(N)$, so $(S)$ cannot be included in Corollary 3.

\section{References}

S. A. Amitsur (1957), 'A generalization of Hilbert's Nullstellensatz', Proc. Amer. Math. Soc. 8, 649-656, MR 19, 384.

V. A. Andrunakievic and Ju. M. Rjabuhin (1968), 'Rings without nilpotent elements and completely prime ideals', Soviet Math. Dokl. 9, 565-568, MR 37, 6320.

Ram Awtar (1973), 'A remark on the commutativity of certain rings', Proc. Amer. Math. Soc. 41, 370-372, MR 48, 6184.

M. P. Drazin (1955), 'Engel rings and a result of Herstein and Kaplansky', Amer. J. Math. 77, 895-913, MR 17, 1048.

R. N. Gupta (1970), 'Nilpotent matrices with invertible transpose', Proc. Amer. Math. Soc. 24, 572-575, MR 40, 5628 .

I. N. Herstein (1963), 'A remark on rings and algebras', Mich. Math. J. 10, 269-272, MR 28, 2129.

N. Jacobson, Structure of Rings (rev. ed., Amer. Math. Soc. Colloq. Publ., vol. 37, Amer. Math. Soc., Providence, R.I.).

T. P. Kezlan (1966), 'Rings in which certain subsets satisfy polynomial identities', Trans. Amer. Math. Soc. 125, 414-421, MR 36, 211.

Department of Mathematics,

University of Missouri - Kansas City,

Kansas City, Missouri 64110,

U.S.A. 